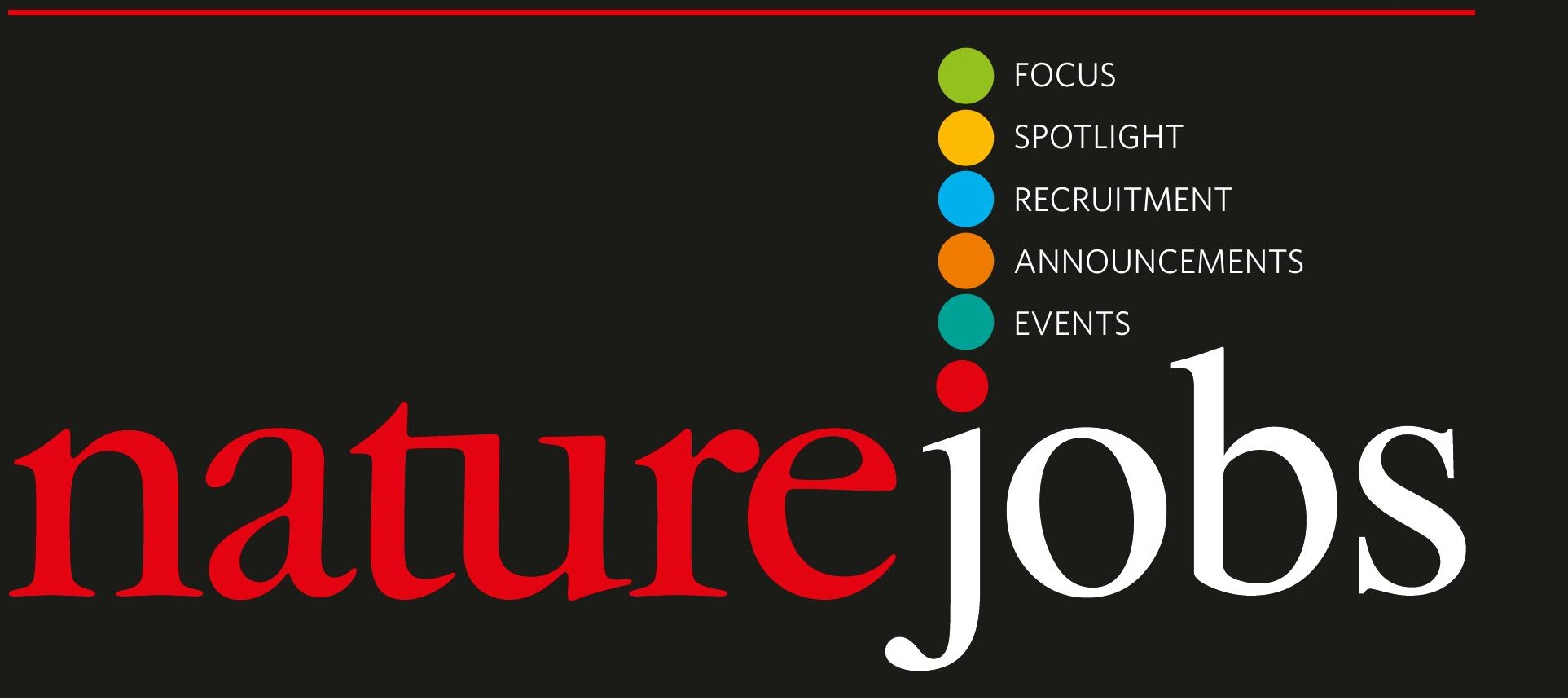

\title{
They'll see you now
}

If you're like most people, waiting for a performance review is akin to sitting in reception at the dentist's. You hope your appointment isn't going to result in any pain, but you expect the worst.

Perhaps some scientists aspire to academia in order to avoid such meddlesome interactions, but two UK lab heads say that feedback is a valuable part of the scientific process. The trick, they say, is making these routine check-ups a two-way process. They advise keeping the meetings relatively informal and focused on progress, rather than deficiency.

Kay Davies is head of the University of Oxford's anatomy department, honorary director of the Medical Research Council's Functional Genetics Unit and co-director of the Oxford Centre for Gene Function. She makes appraisals an annual event for the scientists she directs, and says they keep her researchers focused on specific objectives.

Yearly meetings help them to see what sort of research opportunities exist and how to pick up the necessary skills and resources, she adds.

A different strategy is used by Steve Jackson, who heads a lab at the Gurdon Institute of Cancer and Developmental Biology in Cambridge. He conducts informal exit interviews when students and postdocs end their stint. These casual conversations give the young scientists career goals and provide Jackson with valuable feedback about his lab's direction. He has also used the chats to find employees for KuDOS Pharmaceuticals, a biotechnology company that he created in 1997.

Labs all over the world differ in their policy towards such interactions. Some provide few opportunities for appraisals or feedback, others rely on casual conversations, still others apply a more formal framework. But in science, as in dentistry, regular appointments - whether initiated by you or your supervisor - can improve your long-term health.

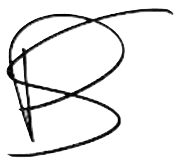

\section{Paul Smaglik, Naturejobs editor}

CONTACTS

Publisher: Ben Crowe

Editor: Paul Smaglik

Marketing Manager: David Bowen

US Head Office, New York

345 Park Avenue South, 10th Floor,

New York, NY 10010-1707

Tel: +1 8009897718

Fax: +18009897103

e-mail: naturejobs@natureny.com

US Sales Manager/Corporations: Peter Bless

Classified Sales Representatives

Tel: +1800989 7718
New York/Pennsylvania/ Latin America: Kelly Roman Midwest USA/Maryland/ NIH: Wade Tucker

East USA/Canada:

Janine Taormina

San Francisco Office

Classified Sales Representative:

Michaela Bjorkman

West USA/West Corp. Canada

225 Bush Street, Suite 1453

San Francisco, CA 94104

Tel: +1415 7813803

Fax: +1 4157813805

e-mail:m.bjorkman@naturesf.com
European Head Office, London

The Macmillan Building,

4 Crinan Street,

London N19XW, UK

Tel: +44 (0) 2078434961

Fax: +44 (0) 2078434996

e-mail: naturejobs@nature.com

Naturejobs Sales Director: Nevin Bayoumi (4978) European Sales Manager: Andy Douglas (4975)

Advertising Production Manager: Billie Franklin To send materials use London address above. Tel: +44 (0) 2078434814

Fax: +44 (0) 2078434996

e-mail: naturejobs@nature.com
Naturejobs web development: Tom Hancock Naturejobs online production: Niamh Shields

European Satellite Office

Patrick Phelan

e-mail:p.phelan@nature.com

Japan Head Office, Tokyo

Chiyoda Building,

2-37 Ichigayatamachi,

Shinjuku-ku,

Tokyo $162-0843$

Tel: +81332678751

Fax: +81332678746

Asia-Pacific Sales Director: Rinoko Asami

e-mail: r.asami@naturejpn.com 